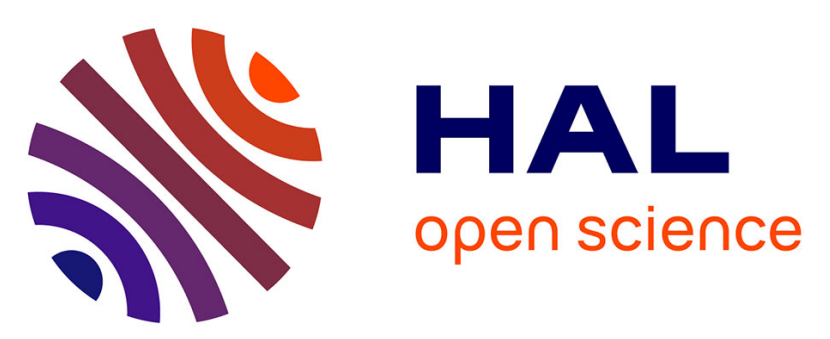

\title{
Identification from measurements of mechanical fields by finite element model updating strategies
}

Patrick Ienny, A. S. Caro-Bretelle, Emmanuel Pagnacco

\section{To cite this version:}

Patrick Ienny, A. S. Caro-Bretelle, Emmanuel Pagnacco. Identification from measurements of mechanical fields by finite element model updating strategies. Revue Européenne de Mécanique Numérique/European Journal of Computational Mechanics, 2012, 18 (3-4), pp.353-376. 10.3166/ejcm.18.353-376 . hal-03237568

\section{HAL Id: hal-03237568 \\ https://hal.mines-ales.fr/hal-03237568}

Submitted on 28 May 2021

HAL is a multi-disciplinary open access archive for the deposit and dissemination of scientific research documents, whether they are published or not. The documents may come from teaching and research institutions in France or abroad, or from public or private research centers.
L'archive ouverte pluridisciplinaire $\mathbf{H A L}$, est destinée au dépôt et à la diffusion de documents scientifiques de niveau recherche, publiés ou non, émanant des établissements d'enseignement et de recherche français ou étrangers, des laboratoires publics ou privés. 


\title{
Identification from measurements of mechanical fields by finite element model updating strategies
}

\author{
A review \\ Patrick Ienny* — Anne-Sophie Caro-Bretelle* \\ Emmanuel Pagnacco** \\ * Ecole des Mines d'Alès \\ Centre des Matériaux de Grande Diffusion \\ 6 Avenue de Clavières, F-30319 Alès cedex \\ Patrick.Ienny@ema.fr \\ ** INSA de Rouen, LMR \\ BP 8, F-76801 St. Etienne du Rouvray cedex
}

\begin{abstract}
Inverse problem resolution methods are widely used in the determination of material behaviour. The optimisation of the parameters, as inputs into a well-defined system, is obtained from observed outputs such as kinematic field measurements. The aim of this paper is to summarize the research concerning one inverse method, Finite Element Modelling Updating, based on the use of these field measurements. This method is based on a combination of three components, described in the following three sections. First we present the optical field measurements applied to multi-axially loaded objects, together with their performances. Then we outline the use of Finite Element Modelling for achieving a correlation between numerical fields and their experimental counterparts. Finally we describe the identification process, together with cost functions, minimisation procedure and model validation analysis.

RÉSUMÉ. Les méthodes de résolution de problèmes inverses sont notamment utilisées dans la détermination de comportements matériaux : l'identification de paramètres, comme variables d'entrée d'un problème bien posé, est obtenue sur la base des données en sortie telles que les mesures de champ. L'objectif de cet article est de produire une synthèse des recherches relative à l'utilisation d'une méthode d'inversion dite de recalage d'un modèle par éléments finis, lorsque celle-ci se base sur l'exploitation de ces mesures de champs. Cette méthode associe trois éléments principaux : les mesures de champs par voie optique, la modélisation des essais par éléments finis ainsi que les fonctions coûts et procédures d'optimisation.

KEYWORDS: finite elements, parameter identification, inverse problems, full-field measurements.

MOTS-CLÉS : éléments finis, identification de paramètres, problèmes inverses, mesures de champs.
\end{abstract}




\section{Introduction}

In recent decades, experimental devices and methodologies have evolved towards the use of full-field measurements and their introduction into various identification methods. Their main objective is to evaluate constitutive material parameters in a reliable and multi-scale manner. They are all derived from well-known principles of continuum mechanics. The Constitutive Equation Gap Method (Geymonat et al., 2003) is based on the minimisation of a function that expresses the gap in the constitutive equation; the Equilibrium Gap Method (Claire et al., 2004) minimises equilibrium residuals; the Reciprocity Gap Method (Bui et al., 2004) is based on the Betti reciprocity theorem of linear elasticity applied to two states (the measured field and an arbitrarily chosen adjoining state); the Virtual Field Method (Grédiac, 1989) uses the virtual work principle; and the Finite Element Method, which can be used as an analysis tool to express the gap between measured and simulated fields. This last strategy, called the Finite Element Model Updating (FEMU) method, on which we shall focus in this presentation, can handle complex (3D) loads and geometrical shapes using displacement and load measurements, independently of the type of behaviour, at the structural scale. The mixed method was originally developed by coupling the Finite Element (FE) model of static tests and experimental information such as strains (generally measured at a limited number of points) or displacements measured by Linear Variable Differential Transformer (LVDT). Nowadays, with full-field measurements, the mixed numerical-experimental method opens up a very broad range of experimental investigation possibilities, such as:

- the mechanical properties of structural materials such as composites: the extreme anisotropy and/or non-homogeneous distribution of material properties over the specimen produce non-homogeneous stress and strain fields in the investigated volume;

- new experimental designs: these enable loading conditions to be defined that are much closer to those of practical industrial situations;

- the study of non-homogeneous strain induces a higher strain level: with the homogeneous response obtained using previous standard tests only local information could be used to analyse the strain response, which could result in the necking of the specimen;

- the analysis of multi-scale modelling by using optic and/or electronic microscopy with mechanical tests in-situ.

The use of optical measurements of kinematics fields enables more complex tests to be carried out (multi-axial loading, experiments on structure components and nonlinear material behaviour) where contact-less measurements of the whole strain field are accessible. Major developments in field measurement techniques are linked to Charges Coupled Device (CCD) sensors and generally to progress in photonics and optical components, together with automated image processing techniques. Many field measurement techniques are available (Surell, 2004): geometric methods such as $2 \mathrm{D}$ or $3 \mathrm{D}$ Digital Image Correlations, fringe projection, deflectometry, grid and 
Moiré methods; and interferometric methods like Moiré interferometry, speckle interferometry and shearing interferometry. We shall now return to the description of these methods, their applications and their expected performances.

As optical methods (apart from 3D-Digital Image Correlation (DIC)) are necessarily one or two-dimensional, in-plane or out-of plane measurements are generally performed using thin specimens. These experiments are related to $2 \mathrm{D}$ simulations (mostly plane stress assumption) to minimise calculation time. Field measurements are often linked to global ones: for example applied stress or loading and displacement measurements. They supply a higher degree of freedom in the test together with more flexible processing. They can also include new boundary conditions such as parasitic effects induced by jaw sliding, bending effects, etc. In the second part, we will present the terms for particular utilisations of FE modelling. Field measurements also enable the design of test geometry in order to increase the sensitivity of the material response to the parameter set of the behaviour. It is thus possible to perform tests in situ, similar to industrial environments. In addition, the non-intrusive and contact-less nature of optical methods enables kinematic measurements that would be too difficult to access by other methods (tiny test geometry, protected site measurements). Nevertheless, once again, the test must be modelled in order to be properly simulated: measurements of realistic boundary conditions, exact geometry of specimens and experimental design are not always carried out and require generally additional assumptions. Various studies can be classified according to the number of identified parameters, which are linked to the complexity of the laws applied (anisotropy, non-linearity, temperature dependence, speed, damage etc.), to the tests conducted (traction, compression, bending) and to the minimisation methodology applied. Indeed, any iteration of the finite element model updating process is very processing-time consuming, as each evaluation needs a complete finite element analysis. Several possibilities have been explored to minimise the processing time while retaining permanent accuracy of the identified parameters. For non-linear computation, first-order methods (or descent methods), sometimes combined with an evolutionary strategy, are generally preferable to a zeroorder algorithm because of their robustness and convergence speed. In the third part we will present the types of identified material behaviour. Finally, we will discuss the optimisation procedures used and their identification reliability.

\section{Kinematic fields, measurements and sensitivity}

\subsection{Optical full-field measurement methods and applications}

The principles of the optical full-field measurement (OFFM) methods are now well-known and have been used for several decades. Moreover, recent developments of optical components and improvements in data processing have led to a tremendous expansion in the use of whole-field measurement techniques (speckle interferometry and digital image correlation amongst others). 
Given the numerous optical techniques for measuring kinematic fields, the analyst is often confronted with the difficulty of selecting the most appropriate method for the target application. This selection is based on knowledge of the mechanical experiment (the measurand, the range of measurement, the dynamic of the observed phenomena) and the performances of the optical techniques. Associated to the choice of optical components and data processing, we can advance some basic parameters which play a key role in the selected method:

1) the spatial resolution which is the smallest distance over which two measurements are independent: for the measurement of a local strain or the characterisation of a distribution of displacement or deformation, this size determines the minimal wavelength or the cut frequency of the detectable local gradients;

2) the sensitivity of the measured entity (measurand), which is the smallest measurable value, i.e. which can be distinguished from background noise: it is generally associated to the standard deviation of the noise measurement and is obtained either by comparison with successive field measurements or using a reference configuration.

An essential stage, and which has an impact on both these parameters, is the postprocessing. Both the subset size (for random encoding) and the pitch (when the surface is encoded by structured light or a grid pattern attached to the surface) define the spatial resolution. This entity is generally chosen as a satisfactory compromise between sensitivity and detail lost due to over-smoothing of the data. So, in practice, the operator is confronted with the inter-dependency of these two parameters for a given optical method and its associated testing configuration (choice and position of optical components and specimen under study, etc.).

While these essential characteristics should be mentioned in all the studies exploiting OFFM methods, another parameter, the measurement uncertainty, can indicate the possibility of exploiting a given method in another laboratory or industrial application, for example. Although it is almost never discussed in research studies, measurement uncertainty is an essential element in the expansion of whole-field methods.

\subsection{Uncertainty of the data}

\subsubsection{Sources of error}

Optical full-field measurement methods provide huge amounts of data whose relevancy is not fully controlled due to a lack of standards and metrology. Among the main sources of errors, for some applications the optical components introduce distortion effects. These systematic errors involve some post-processing using cameras and experimental modelling. But the most significant source of systematic error is the out-of-plane error due to the Poisson contraction of the specimen. Only 3D correlation such as stereo-correlation can avoid this artefact. Recently, some studies have begun to appear following the example of the ESPI method regarding uncertainty evaluation, with the so-called law of propagation of uncertainties (ISO 0.7-0.20): the strain and strain rate capabilities are evaluated by considering the misalignment of the CCD, 
the magnification factor and the coordinates of points between which the relative displacement is measured (Montay et al., 2007). Other systematic errors are introduced in the algorithms themselves, such as the interpolation functions for evaluating the noninteger location using the correlation methods or shape functions which approximate the displacement field. Generic interpolation and approximation algorithms (using linear, cubic and spline weight functions) eliminate high-frequency information (which could be the source of local physical phenomena) through implicit filtering, and introduce fundamental assumptions concerning the form of experimental data (Silva et al., 2007), (Giton et al., 2006).

\subsubsection{Test geometry and boundary conditions}

From an experimental point of view, it is very important to collect accurate data on the design of the test setup, the optical components and the material orientations. Special care should be taken in the experiment to prevent misalignment of the camera along the axis of loading and its specific orientation to the plane of the specimen.

Some other parameters required are the measurement of the specimen and the support geometries, the loading conditions and their correspondence with measured fields (Giton et al., 2006). An interesting approach is to evaluate the boundary conditions using a post-processing operation. For example, finding the exact location of the load point consists in minimising the degree of statistical correlation between the variations in the displacement fields due to an increment of different loading configurations in the FE modelling.

\section{Finite element method}

\subsection{Principles of the finite element method}

Let $\mathcal{B}$ be a solid occupying, in a reference configuration, a domain $\Omega_{0} \in \mathbb{R}^{3}$ with its boundary (sufficiently regular) denoted by $\partial \Omega_{0}$ and let $\mathbf{n}_{0}$ be the corresponding outward normal unit vector. When $\mathcal{B}$ is subject to loads, it undergoes a deformation motion specified by the displacement field $\mathbf{u}$ in $\Omega_{t}$ which is the deformed counterpart of $\Omega_{0}$ with $\mathbf{n}$ the outward normal unit vector to $\partial \Omega_{t}$. In continuum mechanics, the principle of virtual work states that: :

$$
\begin{aligned}
W^{*}= & \int_{\Omega_{t}} \mathbf{D}^{*}: \boldsymbol{\sigma} \mathrm{d} V+\int_{\Omega_{t}} \mathbf{u}^{*} \cdot \rho \cdot \ddot{\mathbf{u}} \mathrm{d} V-\oint_{\partial \Omega_{t}} \mathbf{u}^{*} \cdot \boldsymbol{\sigma} \cdot \mathbf{n} \cdot \mathrm{d} S=0 \\
& \forall t, \mathbf{u}^{*} \text { sufficiently regular }
\end{aligned}
$$

where $\boldsymbol{\sigma}$ is the Cauchy stress tensor, $\rho$ is the material density, $\mathbf{u}^{*}$ refers to a virtual motion and $\mathbf{D}^{*}=\frac{1}{2}\left(\nabla \mathbf{u}^{*}+\nabla \mathbf{u}^{* T}\right)$ to a virtual strain. The current stress state can be related to a strain state (defined from $\mathbf{u}$ ) by choosing an appropriate constitutive law representative of the material behaviour. 
Finite Element Modelling (FEM) (Bathe, 1996) is a numerical strategy which expresses an approximate form of the principle [1] by introducing a representation of the (real and virtual) displacement field in a finite-dimensional space (spanned by the finite element shape functions), leading to:

$$
W^{*}=\mathbf{U}^{* T} \mathbf{F}_{\text {int }}(\boldsymbol{\theta}, \mathbf{U}, \dot{\mathbf{U}}, \ldots)+\mathbf{U}^{* T} \mathbf{M} \ddot{\mathbf{U}}-\mathbf{U}^{* T} \mathbf{F}_{\text {ext }}=0 \quad \forall t, \mathbf{U}^{*}
$$

In this equation, $\mathbf{M}$ is the global mass matrix of the system, $\mathbf{F}_{\text {ext }}$ is the vector of generalised nodal forces and $\mathbf{F}_{\text {int }}$ a vector of internal forces related to the current stress state and possibly to its history. Vector $\mathbf{U}$ groups together the unknown degrees of freedom (dof) introduced by the FEM discretisation (usually nodal displacements). Equation [2] leads to the global, discretised, equilibrium:

$$
\mathbf{M U ̈}=\mathbf{F}_{\text {ext }}-\mathbf{F}_{\text {int }}(\boldsymbol{\theta}, \mathbf{U}, \dot{\mathbf{U}}, \ldots)
$$

Moreover, when inertial effects can be neglected, the discretised equilibrium can be reduced to

$$
\mathbf{F}_{\text {int }}=\mathbf{F}_{\text {ext }} \quad \text { or } \quad \text { K.U }=\mathbf{F}_{\text {ext }}
$$

with $\mathbf{K}$ a secant stiffness matrix. In the context of small perturbations with linear material behaviour, $\mathbf{K}$ will be independent of $\mathbf{U}$.

\subsection{Finite element analysis and the "direct problem"}

The most common problem in continuum mechanics consists in determining the displacement, strain and stress fields over the time $t$, given the initial geometry of the solid, the constitutive parameters and a set of boundary conditions. It is usually referred to as direct or forward in the literature, and is well-posed and qualified for a suitable choice of the set of boundary conditions. For this, each of the boundaries is decomposed into two disjoint open parts denoted by $S_{0 u}, S_{0 r}, S_{t u}$, and $S_{t r}$ such that $\partial \Omega_{0}=S_{0 u} \cup S_{0 r}$ and $\partial \Omega_{t}=S_{t u} \cup S_{t r}$ with a displacement $\overline{\mathbf{u}}$ prescribed on $S_{t u}$ and a surface density force $\overline{\mathbf{f}}_{\mathrm{S}_{\mathrm{r}}}=\boldsymbol{\sigma} . \mathbf{n}$ applied on $S_{t r}$ (which could be imposed as null over a portion of it). This enables the definition of a set of kinematically admissible (K.A.) displacement fields over which u must be found to satisfy

$$
\begin{aligned}
W^{*}= & \int_{\Omega_{t}} \mathbf{D}^{*}: \boldsymbol{\sigma} \mathrm{d} V+\int_{\Omega_{t}} \mathbf{u}^{*} \cdot \rho \cdot \ddot{\mathbf{u}} \mathrm{d} V-\oint_{S_{t_{r}}} \mathbf{u}^{*} \cdot \overline{\mathbf{f}}_{\mathrm{s}_{\mathrm{r}}} \cdot \mathrm{d} S-\oint_{S_{t u}} \mathbf{u}^{*} \cdot \overline{\mathbf{f}}_{\mathrm{s}_{\mathrm{u}}} \cdot \mathrm{d} S=0 \\
& \forall t, \mathbf{u}^{*} \text { sufficiently regular }
\end{aligned}
$$

where $\overline{\mathbf{f}}_{\mathrm{s}_{\mathrm{u}}}=\boldsymbol{\sigma}$.n corresponds to the unknown surface density applied to $S_{t u}$ required to impose the prescribed displacement $\overline{\mathbf{u}}$ on $S_{t u}$. A remedy to this situation consists in choosing the virtual displacement field $\mathbf{u}^{*}$ K.A. 0 to obtain the usable form for the direct problem

$$
\begin{aligned}
W^{*}= & \int_{\Omega_{t}} \mathbf{D}^{*}: \boldsymbol{\sigma} \mathrm{d} V+\int_{\Omega_{t}} \mathbf{u}^{*} \cdot \rho \cdot \ddot{\mathbf{u}} \mathrm{d} V-\oint_{S_{t_{r}}} \mathbf{u}^{*} \cdot \overline{\mathbf{f}_{\mathrm{r}}} \\
& \forall \mathbf{u}^{*} \text { sufficiently regular and for } \mathbf{u} \text { K.A. }
\end{aligned}
$$


Since closed-form solutions to these direct problems are only available in very particular cases, the FEM method can be used in a numerical analysis approach (leading to finite element analysis or FEA) by solving [3] or [4] for $\mathbf{U}$ subjected to the discretised constraint $\mathbf{C} . \mathbf{U}=\overline{\mathbf{U}}$, with $\mathbf{C}$ a boolean (selection) matrix. This step does not deal with the discretised reaction loads $\mathbf{R}=\mathbf{C} . \mathbf{F}_{\text {ext }}$ involved in $\mathbf{F}_{\text {ext }}$ and corresponding to $\overline{\mathbf{f}}_{\mathrm{s}_{\mathrm{u}}}$. But these reaction loads can be recovered in a second step, by evaluating $\mathbf{F}_{\text {ext }}$ from [3] or [4], knowing $\mathbf{U}$. Most of the time, this type of direct analysis is used iteratively in the inverse problems presented in the following parts, by choosing initial parameters and comparing the discretised fields obtained to the experimental ones, until convergence.

\section{Parameter identification using finite element model updating and full field measurements}

\subsection{Theory}

One of the methods used to identify the parameters involved in mechanical tests is known as Finite Element Model Updating. The principle of this technique compared to the classical (analytic) identification strategy is presented in Figure 1. It consists in building a finite element model of the mechanical test under study using data about geometry and boundary conditions, represented by $\varpi$. Experimental data are mostly accessible at discrete intervals (e.g. time or load steps) and the FEM generated data which must be transformed inside the observation space to be compared to experimental data. The observed response from the experiments is denoted $\mathbf{m}(t)$ at each step of time $t \in[0, T]$ (displacements, strains, forces etc.), the corresponding information from the model is denoted $\mathbf{h}_{\varpi}(\boldsymbol{\theta}, t)$ where $\boldsymbol{\theta}$ is the set of parameters to extract (material parameters, stiffness, local load etc.) and $\boldsymbol{\theta}^{(0)}$ is the initial estimate of $\boldsymbol{\theta}$ (necessary entities to start the optimisation scheme). The parameters $\theta$, the input and output variables $\mathbf{m}(t)$ and $\mathbf{h}_{\varpi}(\boldsymbol{\theta}, t)$ are stored in columns:

$$
\begin{aligned}
& \mathbf{m}(t)=\left[m_{1}(t), m_{2}(t), \ldots, m_{N}(t)\right] \\
& \mathbf{h}_{\varpi}(\boldsymbol{\theta}, t)=\left[h_{1_{\varpi}}(\boldsymbol{\theta}, t), h_{2_{\varpi}}(\boldsymbol{\theta}, t), \ldots h_{N_{\varpi}}(\boldsymbol{\theta}, t)\right] \\
& \boldsymbol{\theta}=\left[\theta_{1}, \theta_{2}, \ldots, \theta_{p}\right]
\end{aligned}
$$

where $N$ and $p$ respectively denote the total number of measurements and the number of parameters to be identified.

Depending on the choice of measurable quantities used for identification, a very wide range of situations can be considered. In fact, it is desirable, but by no means necessary, that field measurements should be used. The FEMU technique, because of its principle, may be applied to any kind of over-determined data. 


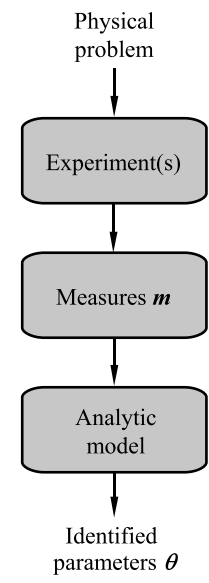

(a) analytic identification

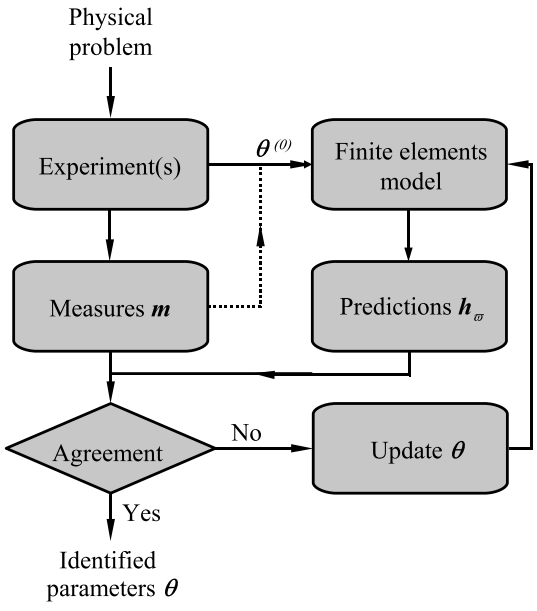

(b) finite element model updating identification

Figure 1. Schematic view of analytic and FEMU identification

When output data are chosen for benchmarking, there is another choice concerning the criterion to be optimised. This criterion can either be algebraic, such as the least square criterion (usual or weighted), or probabilistic, such as likelihood. The first criterion implies looking for the parameters that tend to minimise the distance between $\mathbf{m}(t)$ and $\mathbf{h}_{\varpi}(\boldsymbol{\theta}, t), \forall t \in[0, T]$ among all the possible $\boldsymbol{\theta}$ factors. This general minimisation problem can be written by means of a functional $: J: P \rightarrow \mathbb{R}$ :

$$
\min _{\boldsymbol{\theta} \in U} J(\boldsymbol{\theta})=\min _{\boldsymbol{\theta} \in U} \sum_{t=0}^{T} \frac{1}{2} \mathbf{j}_{\varpi}(\boldsymbol{\theta}, t)^{T} \mathbf{V} \mathbf{j}_{\varpi}(\boldsymbol{\theta}, t)
$$

where $P$ is a part of $\mathbb{R}^{p}$ and

$$
\mathbf{j}_{\varpi}(\boldsymbol{\theta}, t)=\mathbf{m}(t)-\mathbf{h}_{\varpi}(\boldsymbol{\theta}, t)=\left[j_{1_{\varpi}}, j_{2_{\varpi}}, \ldots, j_{N_{\varpi}}\right]
$$

with $j_{i_{\varpi}}: P \times[0, T] \rightarrow \mathbb{R}^{N} \forall i \in[0 \ldots N]$ and $\mathbf{V}$ is a $N \times N$ matrix. In a large range of applications the convexity of the function $J$ with respect to $\theta$ is not demonstrated and the question of the existence and uniqueness of the solution of [8] is an open problem.

The second criterion, based on a minimum variance estimator, implies that the measurement errors $\boldsymbol{e}(t)$ in

$$
\mathbf{m}(t)=\mathbf{h}_{\varpi}(\boldsymbol{\theta}, t)+\mathbf{e}(t)
$$


follow a probability law. The optimal parameters minimise the following quadratic expression:

$$
\min _{\boldsymbol{\theta} \in P} J(\boldsymbol{\theta})=\min _{\boldsymbol{\theta} \in P} \sum_{t=0}^{T} \frac{1}{2} \mathbf{j}_{\varpi}(\boldsymbol{\theta}, t)^{T} \mathbf{V} \mathbf{j}_{\varpi}(\boldsymbol{\theta}, t)+\left(\boldsymbol{\theta}^{(0)}-\boldsymbol{\theta}\right)^{T} \mathbf{W}\left(\boldsymbol{\theta}^{(0)}-\boldsymbol{\theta}\right)
$$

where $P$ is a part of $\mathbb{R}^{p}$ and $\mathbf{j}_{\varpi}(\boldsymbol{\theta}, t)$ is described in [9], $\boldsymbol{\theta}^{(0)}$ is an initial guess for the parameter $\boldsymbol{\theta}$ and $\mathbf{V}, \mathbf{W}$ are $N \times N$ matrices. This criterion is widely used in medical applications (Hendricks, 1991; Oomens et al., 1993; Van Ratingen, 1994) and FEMU structural dynamics (Collins et al., 1974; Friswell et al., 1995).

Nevertheless, both of these minimisation problems are solved by an iterative procedure: find $\boldsymbol{\theta}$ solution of [8] (or [10]) such that

$$
\boldsymbol{\theta}^{(k+1)}=f\left(\boldsymbol{\theta}^{(k)}\right) \text { and } \boldsymbol{\theta}=\lim _{k \rightarrow+\infty} \boldsymbol{\theta}^{(k)} .
$$

The choice of $f$ depends on the chosen resolution algorithm (see section 4.3).

\subsection{Finite elements and full-field measurements}

The FEMU strategy coupled with full-field measurements is especially suitable for non-homogeneous material responses. This explains why materials which exhibit heterogeneous behaviour, homogeneous materials with particular geometries that generate localisation phenomena, or bi-materials (Burczynski, 2003; Cugnoni et al., 2006; Latourte et al., In Press) are usually used as applications.

Rather than giving an inventory of optical methods and their performances, which the reader can find in the literature (Surell, 2004), below we present the applications of these techniques when combined with the FEMU method. Two fundamental orientations need to be considered: on the one hand, more or less local phenomena with high strain gradients, and on the other hand larger-scale observations.

Logically, after the standard methods presented above, full-field displacement or strain measurements were applied to macroscopic-scale quasi-homogeneous tests. All the optical methods are used to determine the in-plane or out-of-plane displacement field (Van Ratingen, 1994; Padmanabhan et al., 2007; Bruno et al., In Press; Genovese et al., 2006b). Geometrical methods are also used (Projection Moiré, 2D or 3D Digital Image Correlation and Video-Tracking, which is based on markers attached to the surface of the specimen) as are Interferometric methods (Moiré Interferometry, Speckle Interferometry and shearing interferometry, which gives the full-field shear strain). In most of the studies, Standard Planar Bi-axial tension tests (in-plane-measurement) or inflation tests (out-of-plane measurement) are applied to thin specimens so that a state of plane stress generally prevails. In addition, the deformation is assumed to be transversely isotropic so only one lateral direction can be used. However, there are 
some examples of 3D-problem resolution studies whose aim is to estimate boundary conditions: both the magnitude and distribution of the contact forces between assembled 3D components have been evaluated using forward resolution (Padmanabhan et al., 2007). An interesting investigation concerned the optimisation of the geometry and/or loading conditions to generate a displacement field more sensitive to certain material parameters (Bruno et al., In Press): for example, to generate a displacement field more sensitive to the value of the Poisson coefficient with a bi-axial flexure test on disk specimen, the circular previously designed loading fixture was converted to a three-point support. It's necessary to note that the analysis of the experimental setup becomes more complex and can only be performed numerically using a computer. It is necessary to use some finite element codes and the improvement of the test is similar to the procedure used for shape optimisation.

At the same observed scale, some studies have focused on more heterogeneous tests where the spatial resolution still enable the measurement of strain gradients. Open-hole tests on thin material shapes or similar experiments are generally used (Mahnken et al., 1996; Lecompte et al., 2006; Molimard et al., 2005). The same approach is used for Moiré hole drilling analysis: the inverse or backward problem requires the observed displacement fields and knowledge of the elastic material properties to evaluate the boundary conditions in the form of applied principal stresses (Cardenas-Garcia et al., 2006). Other studies focus on more local phenomena like necking, which appears during uni-axial tension tests, or high strain gradients for Compact Tension tests, tear strength tests and tensile tests on bi-material samples (Mahnken et al., 1996; Forestier et al., 2002; Giton et al., 2006; Latourte et al., In Press). In every case the resolution (or signal/noise ratio) increases with the strain level suggesting that identification is more accurate for plastic behaviour (strain more than 1) however the strain gradient become a significant source of errors in particular in the neighbourhood of interfaces between two materials and in the vicinity of singularity. So, in every case, the displacement measurements are always filtered using calibrated functions (splines or the weighted sum of a proper set of orthogonal polynomials). Experimental displacements are generally extracted at the position of the integration points in the FE model and in cases where the strain fields are introduced in the cost-functions, numerical and experimental strains are calculated using the same FEM-routine. For these last two families of studies, optical methods are improved to guarantee the best resolution: notably to increase the sensitivity of interferometric methods, the phase variations due to the displacements are measured by applying a temporal phase shifting algorithm.

At the microscopic scale mechanical tests are performed either in the chamber of a Scanning Electron Microscope (SEM) or in front of a camera with a microscope lens (Hoc et al., 2003; Kajberg et al., 2004b; Cugnoni et al., 2006). In all cases, the optical method is generally combined with a tracking method based on contrast change or Digital Image Correlation. These techniques, which are based on the analysis of an acquired sequence of images after the test, enable dynamic tests such as microscopic high-speed photography to be performed (Kajberg et al., 2004b). Work on SEM approaches concerns the mechanical properties at the characteristic size of the 
micro-structure of the material. Example applications are the study of a solder joint used to interconnect electronic components or the analysis of a polycrystal to obtain an accurate description of the mechanical behaviour of the constituent grains so as to improve polycrystalline models. Microextensometry can be combined with the determination of the local lattice orientation, which is based on Electron back-scattering diffraction (ESBD) techniques (Hoc et al., 2003).

Figure 2 shows some examples of specimens with non-standard shapes used to produce heterogeneous mechanical fields. These samples need to be discretised in order to apply FEA. The dof number is reasonably low so each resolution of the direct problem is low cost in terms of computation. Numerical tests are usually run on a single computer, so 2D simulations with axisymmetric or strain/stress plane assumptions are performed. However (Burczynski, 2003) performed 3D elastic identification simulation on a human pelvis. The high number of dof, associated with the use of an evolutionary algorithm (see section 4.3.2) necessitated parallel computation processes.

\subsection{Cost functions and minimisation procedure}

The inverse problem usually consists in identifying $\boldsymbol{\theta}$ from measured data. In the literature, in function of the application, various forms for the function $\mathbf{j}$ in [8] or [10] and for the weighted matrices $\mathbf{V}$ and $\mathbf{W}$ can be found. For a least-square criterion, the weighted matrix are used as a normalisation if the measured quantities are of different physical dimensions, the impact of each quantity has to be tuned in order to obtain a sum of squares of the same magnitude.

A large part of the inverse problem using full-field measurements is devoted to the identification of material properties. Full-field measurements give experimental data which are available either in $\Omega_{t}$ or on $\partial \Omega_{t}$ and the mechanical formulation of the problem has to be adapted in order to accommodate part or all of the available experimental information. If we denote the measured quantities as $\hat{\bullet}$, it will be possible, depending on the available or selected experimental information, to impose some conditions such as $\mathbf{U}(t)=\hat{\mathbf{U}}(t)$ in $\Omega_{t}$ or $\mathbf{U}(t)=\hat{\mathbf{U}}(t)$ on $\partial \Omega_{t}$ or on $S_{t u}$ with $\mathbf{F}_{\text {ext }}(t)=\hat{\mathbf{F}}(t)$ in $\Omega_{t}$ or $\mathbf{F}_{\text {ext }}(t)=\hat{\mathbf{F}}(t)$ on $\partial \Omega_{t}$ or on $S_{t r}$ for the inverse problem.

\subsubsection{Cost functions}

The first (historical) objective function is based on the experimental availability of all the displacements $\hat{\mathbf{U}}$ and $\hat{\mathbf{F}}$ in $\Omega_{t}$

$$
\mathbf{j}_{\varpi}(\boldsymbol{\theta}, t)=\hat{\mathbf{F}}(t)-\mathbf{F}_{\text {int }}(\boldsymbol{\theta}, t) \forall t \in[0, T] \text { and } \mathbf{V}=\mathbf{I}
$$

leading to the so-called (direct) "force balance method" (also known as the "input residual method" or "equilibrium method") (Kavanagh et al., 1971; Friswell et al., 1995; Pagnacco et al., 2007). In [12] I denotes the identity matrix. Since $\mathbf{F}_{\text {int }}$ is evaluated in this expression from the knowledge of $\mathbf{U}=\hat{\mathbf{U}}$ in $\Omega_{t}$, the "direct problem" is never solved in this method. Evaluating [12] is thus very efficient from a computational point of view, and necessitates only an FE model, not an FEA. 


\begin{tabular}{|c|c|c|}
\hline $\begin{array}{l}\text { (a) Specimen shape of ref. } \\
\text { (Lecompte et al., 2005; } \\
\text { Mahnken et al., 1997; Moli- } \\
\text { mardet al., 2005) or (Pag- } \\
\text { nacco et al., 2005) without } \\
\text { hole }\end{array}$ & $\begin{array}{l}\text { (b) Specimen shape of } \\
\text { ref. (Kajberg et al., 2004a; } \\
\text { Mahnken, 1999) }\end{array}$ & $\begin{array}{l}\text { (c) Specimen shape of ref. } \\
\text { (Kajberg et al., 2004b; } \\
\text { Mahnken, 2000) }\end{array}$ \\
\hline $\begin{array}{l}\text { (d) Specimen shape of ref. } \\
\text { (Meuwissen, 1998) }\end{array}$ & $\begin{array}{l}\text { (e) Specimen shape of } \\
\text { ref. (Meuwissen, 1998; } \\
\text { Meuwissen } \text { et al., 1998) }\end{array}$ & $\begin{array}{l}\text { (f) Specimen shape of ref. } \\
\text { (Giton et al., 2006) }\end{array}$ \\
\hline $\begin{array}{l}\text { (g) Specimen shape of ref. } \\
\text { (Mahnken et al., 1996) }\end{array}$ & $\begin{array}{l}\text { (h) Specimen shape of ref. } \\
\text { (Meuwissen, 1998) }\end{array}$ & $\begin{array}{l}\text { (i) Specimen shape of ref. } \\
\text { (Lecompte } \text { et al., 2007) }\end{array}$ \\
\hline
\end{tabular}

Figure 2. Specimen shapes

Moreover, if the parameterisation of the problem is linear in $\boldsymbol{\theta}$ (for example the bulk and shear moduli, i.e. $\boldsymbol{\theta}=[K, G]$, in a problem devoted to the identification of isotropic material properties), the minimisation problem associated to this objective function is an over-determined system of equations of the form (Kavanagh et al., 1971): (Kavanagh et al., 1971):

$$
\frac{\partial[\mathbf{K}(\boldsymbol{\theta}) \cdot \hat{\mathbf{U}}]}{\partial \boldsymbol{\theta}} \cdot \boldsymbol{\theta}=\hat{\mathbf{F}}
$$

which can be solved using a (non-iterative) least-square method in only one step, without reference to any starting value $\boldsymbol{\theta}^{(0)}$. Consequently, no assumption about $\boldsymbol{\theta}^{(0)}$ is necessary in this method, nor FEA or iteration.

Another common (but more recent) approach (Collins et al., 1974) to the identification problem is obtained by considering an objective function in which data on 
displacement measurements are retained for the minimisation process while the force response is used as boundary condition:

$$
\mathbf{j}_{\varpi}(\boldsymbol{\theta}, t)=\mathbf{j}_{\varpi \mathrm{u}}(\boldsymbol{\theta}, t)=\hat{\mathbf{U}}(t)-\mathbf{U}_{\varpi}(\boldsymbol{\theta}, t) \text { and } \mathbf{V}=\mathbf{V}_{\mathrm{u}}
$$

leading to the so-called "displacement method" or "output residual method". In this expression, $\mathbf{U}_{\varpi}$ is evaluated through a FEA and is commonly arranged as $\mathbf{U}_{\varpi}=\left[\left(U_{1_{x}}, U_{1_{y}}\right),\left(U_{2_{x}}, U_{2_{y}}\right), \ldots,\left(U_{N_{x}}, U_{N_{y}}\right)\right]$ and $\hat{\mathbf{U}}=$ $\left[\left(\hat{U}_{1_{x}}, \hat{U}_{1_{y}}\right),\left(\hat{U}_{2_{x}}, \hat{U}_{2_{y}}\right), \ldots,\left(\hat{U}_{N_{x}}, \hat{U}_{N_{y}}\right)\right]^{1}$ for a number $N$ of nodal data: this can either be the total number of nodes describing the finite element mesh or a selection of well chosen nodes. This is a decisive advantage of this method over the original force method since measurements are not required over $\Omega_{t}$ but could be limited to a specific area. The misfit minimisation is always performed using iterative methods and any possible non-linearity dependency on $\boldsymbol{\theta}$ does not matter. Moreover, another advantage of this method over the force method is that it seems to be less sensitive to noise measurements, according to a numerical experimentation proposed in reference (Cottin et al., 1984).

Since this method necessitates an FEA, displacement (either theoretical or measured) should usually be prescribed over a portion of the boundary in order to obtain a well posed form for the direct problem. Such a choice may certainly have an impact over the inverse problem solution. However (Pagnacco et al., 2006) proposes another strategy to solve the problem without displacement boundary conditions for the FEA. This strategy handles rigid body motion in the formulation and adds force loads to the set of unknown parameters.

In [13], the function can be normalised (or not) by a suitable choice of weighted matrix. For example, $\mathbf{V}_{\mathrm{u}}=\mathbf{I}$ in (Mahnken et al., 1996), $\mathbf{V}_{\mathrm{u}}=\mathbf{V}_{\mathrm{u}}(t)=$ $\left(\hat{\mathbf{U}}(t)-\mathbf{U}^{(0)}(\boldsymbol{\theta})\right)^{-1} \mathbf{I}$ where upper index $(\bullet)^{(j)}$ refers to iteration number of the identification process (Mahnken et al., 1997) and $\mathbf{V}_{\mathrm{u}}=\mathbf{V}_{\mathrm{u}}(t)=\hat{\mathbf{U}}(t)^{-1} \mathbf{I}$ (Genovese et al., 2006a). Consequently, great variety can be established by choosing the matrix weighting $\mathbf{V}_{\mathrm{u}}$, giving several physical meanings. For example, $J$ described by [13], becomes homogeneous to an energy if $\mathbf{V}_{\mathrm{u}}=\mathbf{K}$ and is near to $J$, described by [12], if $\mathbf{V}_{\mathrm{u}}=\mathbf{K}^{2}$.

In (Lecompte et al., 2007), the authors derive measured displacements to evaluate the strains (axial, longitudinal and shear) which appear in the objective function:

$$
\mathbf{j}_{\varpi}(\boldsymbol{\theta}, t)=\mathbf{j}_{\varpi \epsilon}(\boldsymbol{\theta}, t)=\hat{\boldsymbol{\epsilon}}(t)-\boldsymbol{\epsilon}(\varpi, \boldsymbol{\theta}, t) \text { and } \mathbf{V}=\mathbf{V}_{\epsilon}
$$

where $\boldsymbol{\epsilon}(t)=\left[\left(\epsilon_{1_{x}}, \epsilon_{1_{y}}, \epsilon_{1_{x y}}\right),\left(\epsilon_{2_{x}}, \epsilon_{2_{y}}, \epsilon_{2_{x y}}\right), \ldots,\left(\epsilon_{N_{x}}, \epsilon_{N_{y}}, \epsilon_{N_{x y}}\right)\right], \quad \hat{\boldsymbol{\epsilon}}(t)=$ $\left[\left(\hat{\epsilon}_{1_{x}}, \hat{\epsilon}_{1_{y}}, \hat{\epsilon}_{1_{x y}}\right),\left(\hat{\epsilon}_{2_{x}}, \hat{\epsilon}_{2_{y}}, \hat{\epsilon}_{2_{x y}}\right), \ldots,\left(\hat{\epsilon}_{N_{x}}, \hat{\epsilon}_{N_{y}}, \hat{\epsilon}_{N_{x y}}\right)\right]$ for 2D simulations, $N$ corre-

1. In 2D situations, or as $\mathbf{U}_{\varpi}=\left[\left(U_{1_{x}}, U_{1_{y}}, U_{1_{z}}\right), \ldots,\left(U_{N_{x}}, U_{N_{y}}, U_{N_{z}}\right)\right]$ and $\hat{\mathbf{U}}=$ $\left[\left(\hat{U}_{1_{x}}, \hat{U}_{1_{y}}, \hat{U}_{1_{z}}\right), \ldots,\left(\hat{U}_{N_{x}}, \hat{U}_{N_{y}}, \hat{U}_{N_{z}}\right)\right]$ in 3D situations, with subscripts $(\bullet)_{x},(\bullet)_{y}$ and $(\bullet)_{z}$ referring to the coordinate system. 
sponds to the number of elements in the finite e lement $\mathrm{m}$ esh $\mathrm{r}$ epresentation and $\mathbf{V}_{\epsilon}=\mathbf{V}_{\epsilon}(t)=\hat{\boldsymbol{\epsilon}}(t)^{-1} \mathbf{I}$. A stress formulation is adopted in (Molimard et al., 2005):

$$
\mathbf{j}_{\varpi}(\boldsymbol{\theta}, t)=\mathbf{j}_{\varpi_{\sigma}}(\boldsymbol{\theta}, t)=\hat{\boldsymbol{\sigma}}(t)-\boldsymbol{\sigma}(\varpi, \boldsymbol{\theta}, t) \text { and } \mathbf{V}=\mathbf{V}_{\sigma}
$$

In (Cardenas-Garcia et al., 2006) $\boldsymbol{\sigma}(t)$ corresponds to the maximum in plane shear stress.

Works by (Kajberg et al., 2004a; Cugnoni et al., 2006; Giton et al., 2006) focus the objective function on the force response at each time on the simulation treated as implicit functions of the parameters, while displacement measurements are used only on $\partial \Omega_{t}$ as realistic boundary conditions:

$$
\mathbf{j}_{\varpi}(\boldsymbol{\theta}, t)=\mathbf{j}_{\varpi_{\mathrm{F}}}(\boldsymbol{\theta}, t)=\hat{\mathbf{F}}(t)-\mathbf{F}_{\mathrm{int} \varpi}(\boldsymbol{\theta}, t) \forall t \in[0, T] \text { and } \mathbf{V}=\mathbf{V}_{\mathrm{F}}
$$

In this expression $\mathbf{F}_{\text {int } \varpi}$ is limited to $\partial \Omega_{t}$ and must be evaluated through an FEA, and $\mathbf{j}_{\varpi_{\mathrm{F}}}(\boldsymbol{\theta}, t)$ has two or three components if it is a $2 \mathrm{D}$ or $3 \mathrm{D}$ simulation. Here $\mathbf{V}_{\mathrm{F}}$ is often used to normalise the functional $\mathbf{V}_{\mathrm{F}}=\hat{\mathbf{F}}_{\mathrm{e}}^{-1} \mathbf{I}$, where $\hat{\mathbf{F}}_{\mathrm{e}}(T)$ is the yield force at the end of the simulation, (Kajberg et al., 2004a), or to adjust weights on the misfits between measured and computed quantities. For example, Figure 3 shows a comparison between experimental and simulated displacements (with cost function described in [16]) for a biaxial test on a composite material (Pagano, 2006; Claire et al., 2004).

Lastly, some authors combine the previous objective functions: they accumulate $\mathbf{j}_{\varpi_{u}}, \mathbf{j}_{\varpi_{\epsilon}}$ and $\mathbf{j}_{\varpi_{F}}$, scaling them with the difference between the maximum and the mean value of the actual quantities (Kajberg et al., 2004a). Reference (Padmanabhan et al., 2007) combines [15] and [13]. In (Mahnken et al., 1997; Mahnken, 1999; Mahnken, 2000; Giton et al., 2006), the objective function combines $\mathbf{j}_{\varpi_{u}}$ and $\mathbf{j}_{\varpi_{F}}$ such that $\mathbf{V}_{\mathrm{u}}=w_{u} \mathbf{I}$ and $\mathbf{V}_{\mathrm{F}}=w_{F} \mathbf{I}$ where the weighting factors $w_{u}$ and $w_{F}$ are chosen in such a way that $\mathbf{j}_{\varpi_{u}}^{T}(\boldsymbol{\theta}, t) \mathbf{V}_{\mathrm{u}} \mathbf{j}_{\varpi_{u}}(\boldsymbol{\theta}, t)$ and $\mathbf{j}_{\varpi_{F}}^{T}(\boldsymbol{\theta}, t) \mathbf{V}_{\mathrm{F}} \mathbf{j}_{\varpi_{F}}(\boldsymbol{\theta}, t)$ are of the same order. In (Hoc et al., 2003) $j_{\mathrm{F}}$ and $j_{\epsilon}$ are combined together with $\mathbf{V}_{\mathrm{F}}=w_{F} \mathbf{I}$ and $\mathbf{V}_{\epsilon}=w_{\epsilon} \mathbf{I}$ such that the force contribution, which reproduces the best overall response, is predominant.

Finally, recent works (Latourte et al., In Press) use weighted least square estimates to compute both the stress and elasticity tensor from the displacement measurements and applied loads. A variational approach (Geymonat et al., 2003) minimises a function (namely the "constitutive equation gap"), which is separately convex with respect to the stress and elasticity tensor:

$$
\mathbf{j}_{\varpi}(\boldsymbol{\sigma}, \mathbf{A})=\boldsymbol{\sigma}-\mathbf{A}: \boldsymbol{\epsilon}(\hat{U}) \text { and } \mathbf{V}=\mathbf{A}^{-1}
$$

where $\mathbf{A}$ is the elasticity tensor. 


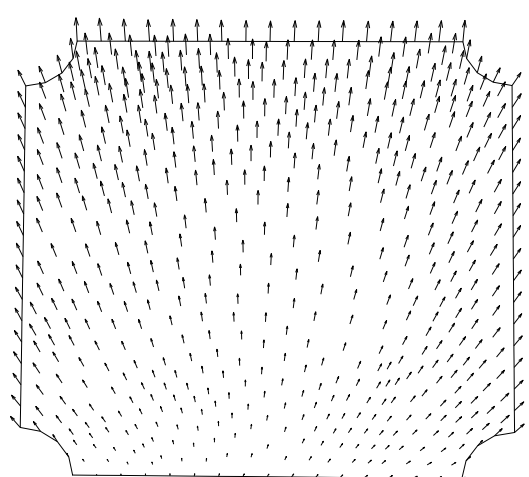

(a) Experimental displacements

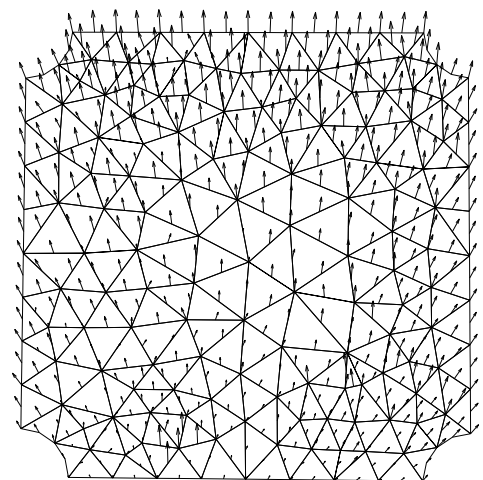

(b) FEMU displacements

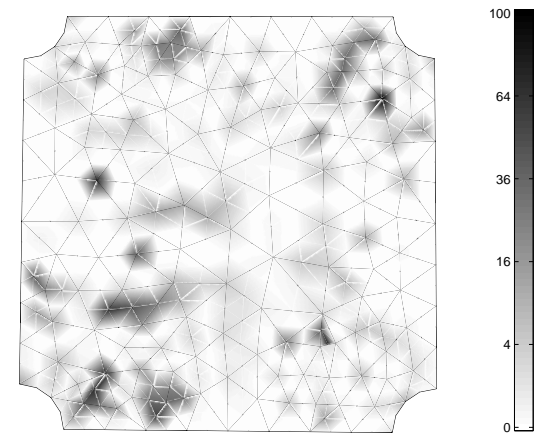

(c) Discrepancies between FEMU and experimental fields displacements (\%)

Figure 3. Displacements field solutions using FEMU strategy for a biaxial test

In the framework of the characterisation of the mechanical properties of biological material, some authors (Oomens et al., 1993; Van Ratingen, 1994; Meuwissen et al., 1998) use the minimum variance criterion and $\mathbf{j}_{\varpi}(\boldsymbol{\theta}, t)=\mathbf{j}_{\varpi_{u}}(\boldsymbol{\theta}, t)$ in [10]. The weighted matrix $\mathbf{V}_{\mathrm{u}}$ and $\mathbf{W}$ are diagonal and related to the available statistics concerning observation errors. Their components are approximately the inverse of the squared expected errors in the displacement and in the initial guess of the parameters, respectively. A larger value of these matrices indicates a greater level of confidence.

In view of the references reported until now in this subsection, we can see that a large proportion of inverse problem statements are concerned with the identification of material properties, but parameters are not limited to this choice. Thus, in references (Lemosse et al., 2003), (Padmanabhan et al., 2007) and (Pagnacco et al., 2005) the authors use [13] and/or [14] to identify both loads applied to the structure and its associated stiffness. And in (Cardenas-Garcia et al., 2006), $\boldsymbol{\theta}$ refers to the applied stress field around the hole in order to evaluate the residual stresses in the material using [13]. 


\subsubsection{Process minimization}

Although the function $J$ in [8] can be minimised by a general unconstrained method (a direct search method such as the simplex method (Kajberg et al., 2004a), (Kajberg et al., 2004b), genetic algorithm (GA) (Burczynski et al., 2004; Bruno et al., In Press) or simulated annealing (SA) (Genovese et al., 2006a; Genovese et al., 2006b), in most general cases special methods adapted to least-square problems are used. Example are the Gauss-Newton method (Oomens et al., 1993; Van Ratingen, 1994; Meuwissen et al., 1998; Forestier et al., 2002; Lecompte et al., 2007) or its alternative the Levenberg-Marquardt method (Molimard et al., 2005; Cugnoni et al., 2006; Giton et al., 2006) which reduces (compared to the Gauss-Newton method) potential instabilities due to ill-conditioning by filtering the lower eigenvalues of the iteration matrix. They are both suitable for low residual cost functions and use the specific form of the gradient of $J$ in their iterative $r$ esolution. All these methods follow this iterative procedure: :

$$
\boldsymbol{\theta}^{(k+1)}=\boldsymbol{\theta}^{(k)}+\rho\left(\boldsymbol{\theta}^{(k)}, \mathbf{d}^{(k)}\right) \cdot \mathbf{d}^{(k)}
$$

where $\rho\left(\boldsymbol{\theta}^{(k)}, \mathbf{d}^{(k)}\right)$ and $\mathbf{d}^{(k)}$ depend on the chosen method. In order to apply these methods one must specify the initial value parameters $\boldsymbol{\theta}^{(0)} \in \mathbb{R}^{p}$.

When the objective function is smooth enough these methods are known to be competitive with respect to their speed resolution. It is possible to modify the GaussNewton method to allow convergence for larger residual problems: this is the Corrected Gauss-Newton method. For these large residue problems the Quasi-Newton approximation (the most famous is the BFGS approximation) does not exploit the least-square nature of the objective function (Gill et al., 2006). A modified version of this Quasi-Newton approximation due to Bertsekas can also be used (Mahnken et $a l .$, 1996). All these gradient methods depend strongly on the initial parameter estimation because of the possible non-uniqueness of the local minimum.

In most general cases, ill-conditioning is a common feature of this non linear leastsquare problem because the underlying mathematical model is often ill-defined. The objective function $J$ can be highly non-smooth and it is possible that the previous methods could not work very well: instability in the iteration matrix $\left(\partial \mathbf{h}_{\varpi} / \partial \boldsymbol{\theta}\right)$ estimated at each step of the analysis is a common feature, whether it is evaluated by an analytical (Mahnken et al., 1996; Mahnken et al., 1997) or a numerical method (i.e. the finite difference approach in (Meuwissen et al., 1998; Giton et al., 2006)).

Direct search methods are one remedy since only the function value is evaluated sequentially and compared to find the optimum. In this framework, the simplex method is a determinist approach which is suitable for a low number of parameters. It consists in evaluating the cost function on a simplex of $\mathbb{R}^{p}$ and moving this simplex with respect to the results obtained. The starting point of these methods is the initial simplex (Kajberg et al., 2004a; Kajberg et al., 2004b). Stochastic algorithms are also direct search methods. In the context of identification, SA and GA are commonly used. They both are based on an analogy with biological systems. SA is based on a thermodynamic process called annealing (Genovese et al., 2006a; Genovese et al., 2006b). GA 
is based on analogies with natural selection strategies from evolution. Both GA and SA have the property of allowing uphill iteration, so the number of iterations does not therefore necessarily decrease monotonically. However, the robustness of these algorithms is obtained at the expense of the computation time since they require further re-analysis to complete the optimisation process. Direct search algorithms, unlike the gradient-based methods, do not require initial estimates but a suitable set of boundaries for the unknowns. Significant improvements in terms of calculation time and the accuracy of the solution can be obtained with respect to the evolution of this boundary set.

In the variational approach [17], the uniqueness of the solution combined with the convexity of the function enables the selection of a relaxation algorithm (Latourte et al., In Press).

In the case of the minimum variance algorithm, the minimisation of the function $J$ in [10] is performed iteratively applying the following scheme:

$$
\boldsymbol{\theta}^{(k+1)}=\boldsymbol{\theta}^{(k)}+\mathbf{D}^{(k+1)}\left(\mathbf{m}(t)-\mathbf{h}_{\varpi}(\boldsymbol{\theta}, t)\right),
$$

where $\mathbf{D}^{(k+1)}$ is a weighting matrix depending on error covariance and iterative matrices.

\subsection{Applications, results and sensitivity}

\subsubsection{Applications to material behaviour}

\subsubsection{Description of material behaviour}

Due to the utilisation of a numerical process to iteratively compute the quantity $\mathbf{h}_{\varpi}$ in [9], a wide variety of material behaviour can be identified using this procedure. From linear isotropic elasticity to hyper-visco-elasticity, there is no restriction.

In the framework of linear elasticity, (Burczynski, 2003; Pagnacco et al., 2005; Cardenas-Garcia et al., 2006; Cugnoni et al., 2006; Genovese et al., 2006a; Latourte et al., In Press) work on isotropic materials while references (Bruno et al., 2002; Lecompte et al., 2006; Molimard et al., 2005; Silva et al., 2007) extend previous computations to anisotropic behaviour. Viscoelasticity can also be identified both for long constant times (see (Le Magorou et al., 2002) for wood-based panel applications) and for short constant times, typical of vibro-acoustic studies (see (Pagnacco et al., 2007) for a polymer application). In the framework of linear elasticity, the group led by Prof. Oomens at Eindhoven University of Technology has undertaken a number of applications of such approaches in the field of bio-mechanics on human skin in vivo (Meijer et al., 1997) or dog skin in vitro (Oomens et al., 1993; Van Ratingen, 1994) assumed to follow an orthotropic elastic law. (Burczynski, 2003) realised a 3D elastic identification simulation on a human pelvis. In all theses cases, the number of parameters to be identified does not exceed 6. References (Kavanagh et al., 1971) and (Genovese et al., 2006a) treat the identification of a hyper-elastic constitutive law (Mooney-Rivlin) from bi-axial tests. In 
(Giton et al., 2006), the same behaviour is used for a PET specimen in a uni-axial test. Up to 7 parameters have to be identified.

A large part of the literature is also dedicated to the identification of the plasticity (or visco-plasticity) of (metallic) material behaviour assuming elastic parameters are known. Among the first studies were (Mahnken et al., 1996) and (Mahnken et al., 1997), where the material was modelled by $J_{2}$ flow theory with non-linear isotropic hardening. This procedure was extended to Gurson's damage modelling in (Mahnken, 1999; Mahnken, 2000). Studies by (Meuwissen et al., 1998; Cugnoni et al., 2006) and (Meuwissen, 1998) deal with the identification of several plasticity models (Von Mises or Hill criteria combined with isotropic or kinematic hardening, etc.) through traction tests on various geometries (notched or drilled specimens). Reference (Kajberg et al., 2004a) performed in-plane traction tests with rate dependent mechanical behaviour to deduce piecewise the linear plasticity and a parabolic hardening model. An extension of this work involving visco-plastic constitutive parameters obtained from high strainrate testing is presented in reference (Kajberg et al., 2004b). In (Forestier et al., 2002) a complete testing of both isotropic and anisotropic viscoplastics involving from 4 to 11 parameters can be found. Finally, at the microscopic scale, (Hoc et al., 2003) identified several hardening laws from a traction test on a polycrystal.

\subsubsection{Identification reliability}

Although the mixed methods enable stringent validation of a constitutive model, there is an indirect relation between errors in the constitutive model and errors in the predicted response. So the objective of some preliminary numerical simulations should be to design suitable experiments for parameter estimation and model validation. In addition, material parameters describing non linear behaviour are not usually compared with parameters deduced from reference tests. This makes the quality of the results difficult to assess.

The stability of the identification results with respect to the chosen mesh is usually checked first. M oreover, for local m ethods, parameter i dentification do es not seem to be affected by a change in the estimate of the initial parameter. The reason is that the solution is usually sought in a restricted space (in which there is a single minimum). For SA based optimisers, distinct optimisation runs have been carried out with different ranges of the set parameters. It seems that material parameter values were rather insensitive to this change (Genovese et al., 2006a), probably due to the high number of data given by full-field measurements.

Assuming stability of the numerical identification, the reliability of identification results has to be assessed. Frequently, authors link the reliability of the identified parameters to the good agreement between experimental and simulated data. Nevertheless, some of them look at the standard deviation of the associated residual. In reference (Meuwissen et al., 1998) the authors validate their results from the similitude between this standard deviation and that of the measurement noise. Unfortunately, this is a global criterion. A study of the first order Gauss Newton approximation: 
$\nabla^{T} \mathbf{j}_{\varpi} \nabla \mathbf{j}_{\varpi}$ is given in (Molimard et al., 2005). An "identifiability factor" defined as the minimum eigenvalue normalised by the maximum eigenvalue allows the authors to discriminate some residue choices. Another approach is that of references (Hendricks, 1991), (Oomens et al., 1993) and (Van Ratingen, 1994), who checked the stability of the results identification by introducing noisy displacements or systematic relative uncertainties (Kajberg et al., 2004a; Kajberg et al., 2004b; Giton et al., 2006). In addition to sensitivity analysis, the correlation between parameters could be studied through the evaluation of the correlation matrix such that: $C_{i j}=\cos \left(\frac{\partial \mathbf{h}_{\varpi}}{\partial \boldsymbol{\theta}_{i}}, \frac{\partial \mathbf{h}_{\varpi}}{\partial \boldsymbol{\theta}_{j}}\right)$. If all off-diagonal elements are close to 1, the parameters are highly correlated and tend to be inaccurate (Forestier et al., 2002).

\subsubsection{Applications in dynamics}

In the field of structural dynamics, reference (Dascotte, 2007) recently made a review of model updating and discussed the expected benefits of the full-field measurements now available in dynamic testing: e.g. interferometry techniques such as ESPI or DSPI, holographic interferometry, and Scanning Laser Doppler Vibrometry (SLDV). These optical measurements can speed up the testing process, with high spatial resolution and without adding masses to the structures under test, which is decisive in many dynamic applications. Many experimental studies have been carried out in order to obtain reliable dynamic quantitative measurements, and the major references in this domain now mainly deal with the step of transforming the optical measurement data into values that are meaningful from a FE point of view (Linet et al., 2001; Michot et al., 2001; Piranda et al., 2003; Simon et al., 2003). For example, references (Michot et al., 2001; Piranda et al., 2003) deal with modal analysis and correlation tools able to handle the large quantity of data given by ESPI (typically several hundred thousand data items). For updating the FEM of a car window, (Linet et al., 2001) used a photogrammetry technique to obtain the correct geometry and validate the resulting numerical model by assimilating operating deflection shapes (measured by pulsed laser ESPI) to normal modes shapes.

More recently, dynamic full-field measurements have been used in inverse mechanical problems to identify material parameters. (Cugnoni et al., 2007) evaluated the elastic properties of multi-layered composite laminates based on extracted mode shapes and natural frequencies of free thin and thick plates. The experimental technique combined a loudspeaker and an SLDV, while the finite element model used an accurate shell element derived from higher order shear deformation theory. The properties were estimated by a nonlinear least-square algorithm applied to an error norm formed by combining several criteria (frequencies, modes shapes and orthogonality results of a modal analysis with anti-resonance localisations). The overall procedure provides accurate identification of the 6 parameters in a single test. (Moreau et al., 2006; Pagnacco et al., 2007) evaluated the possibility of determining the viscoelasticity parameters of isotropic, moderately thick plates from a single experiment. Measurements were performed using an SLDV and an automated impact device. In this work, an extension of the force and displacement method, called a displacement weighted force method, is proposed to handle the frequency response fields in the 
identification p rocedure. The visco-elastic parameters are the real and complex parts of the Young's modulus and the Poisson's ratio, with their frequency dependence but without any parameterisation.

\section{Conclusion}

A common problem of continuum mechanics, the so-called direct or forward problem, refers to the determination of outputs such as displacements, strains and stress fields. For a well defined system, the solution is obtained from knowledge of the inputs such as the geometry of the solid, the constitutive parameters and a set of boundary conditions. The identification of constitutive parameters, boundary conditions or stress distribution is another type of problem, called the inverse or backward problem in the literature. Among the numerical techniques proposed for solving inverse problems, the Finite Element method is the most commonly used. FEMU is a very flexible technique that can be adapted to a wide range of situations and data types. The aim of this article is to review the studies which have applied the Finite Element Model Updating method to identify either the mechanical behaviour of materials or a set of boundary conditions.

In addition, the tremendous expansion in the development of full field displacement and strain measurement provides a large amount of information for the characterisation of the mechanical responses of structures and materials. The possible combination of multi-axially loaded structures with $2 \mathrm{D}$ or 3D-kinematic measurements enables experiments to be designed using conditions that are much closer to those encountered in practical situations, such as many industrial processes. The present paper has given an overview of the applications of the FEMU method in which the experimental data used were full-field measurements.

The FEMU method for material characterization is generally marked by the use of field quantities in experimental situations where homogeneous stress and strain fields can not be obtained. The method has proven to be effective for the characterization of highly anisotropic and non-homogeneous materials and for structural components. But some important tasks must be carried out in the future in order to make this method accurate and exploitable by every potential user.

The accuracy depends of course on many factors including, but not limited to, optical components, image resolution and image processing. Thus, from the experimental point of view, it is very important to gather accurate data on test geometry, the support of the specimen and boundary conditions. So, numerical simulations have to be carried out to investigate the influence of the test setup and to evaluate the maximum sensitivity of the procedure to the unknown parameters. Combined with the experimental data, the resolution of the measurand can satisfy a compromise with the spatial resolution, especially when considering heterogeneous strain fields. One major source of error could be the choice of an inappropriate constitutive model for the material behaviour. Sources of error can also result from direct computation, which may be less 
accurate in the case of a non-linear problem. In all cases, it is difficult to discriminate errors stemming from the measurements from those resulting from numerical processing, undoubtedly because of the lack of a direct relation between these two entities. The cost function and the associated minimisation algorithm seem to play a crucial role in the procedure: the robustness and convergence time of the algorithm, the stability of the solution and the dependency on the initial parameter set are all questions that must be considered in order to achieve a better use of the method.

\section{References}

Bathe K., Finite Element Procedures, Prentice Hall, 1996.

Bruno L., Furgiuele F. M., Pagnotta L., Poggialini A., Stigliano G., “ Elastic Characterization of Orthotropic Plates of Any Shape via Static Testing", International journal of Solids and Structures, In Press.

Bruno L., Furgiuele F., Pagnotta L., Poggialini A., “ A full-field approach for the elastic characterization of anisotropic materials", Optics and Lasers in Engineering, vol. 37, p. 417-431, 2002.

Bui H. D., Constantinescu A., Maigre H., “ Numerical identification of linear crack in 2D elastodynamics using the instantaneous reciprocity gap”, Inverse Problems, vol. 20, p. 993, 2004.

Burczynski T., "Evolutionary Computation in Mechanics”, , IACM Expressions, Bull. for The Int. Assoc. for Comp. Mech., October, 2003. p. 4-9.

Burczynski T., Kuś W., Dugosz A., Orantek P., “ Optimization and defect identification using distributed evolutionary algorithms”, Engineering Applications of Artificial Intelligence, vol. 17, p. 337-344, June, 2004.

Cardenas-Garcia J., Preidikman S., " Solution of the moire hole drilling method using a finiteelement-method-based approach", International Journal of Solids and Structures, vol. 43, $\mathrm{n}^{\circ}$ 22-23, p. 6751-6766, November, 2006.

Claire D., Hild F., Roux S., “ A finite element formulation to identify damage fields: the equilibrium gap method.”, International Journal for Numerical Methods in Engineering, vol. 2, p. 189-208, 2004.

Collins J., Hart G., Kennedy B., “ Statistical identification of structures”, AIAA Journal, vol. 12, $\mathrm{n}^{\circ} 2$, p. 185-190, 1974.

Cottin N., Felgenhauer H., Natke H., " On the parameter identification of elastomechanical systems using input and ouput residuals”, Ingenieur-Archiv, vol. 54, p. 378-387, 1984.

Cugnoni J., Botsis J., Sivasubramaniam V., Janczak-Rusch J., “ Experimental and numerical studies on size and constraining effects in lead-free solder joints", Fatigue Fract. Engng. Mater. Struct., vol. 30, n² 2, p. 387-399, 2006.

Cugnoni J., Gmur T., Schorderet A., "Inverse method based on modal analysis for characterizing the constitutive properties of thick composite plates", Computers and Structures, 2007. In Press.

Dascotte E., “ Model Updating for Structural Dynamics: Past, Present and Future Outlook”, International Conference on Engineering Dynamics (ICED), Carvoeiro, Algarve, Portugal, April 16-18, 2007. cdrom, 12 pages. 
Forestier R., Massoni E., Chastel Y., " Estimation of constitutive parameters using an inverse method coupled to a 3D finite element software", Journal of Materials Processing Technology, vol. 125-126, p. 594-601, 2002.

Friswell M., Mottershead J., Finite element Model Updating in Structural Dynamics, Kluwer Academic Publishers, 1995.

Genovese K., Lamberti L., C. P., “ Mechanical characterization of hyperelastic materials with fringe projection and optimization techniques", Optics and Lasers in Engineering, vol. 44, $\mathrm{n}^{\circ}$ 5, p. 423-442, May, 2006a.

Genovese K., Lamberti L., Pappalettere C., “ Identification of mechanical properties of bovine bones by combining PS-ESPI and global optimization", Speckle06, Nîmes, p. 634108.1634108.7, 2006b.

Geymonat G., Pagano S., “ Identification of mechanical properties by displacement field measurement: a variational approach”, Meccanica, vol. 38, p. 535-545, 2003.

Gill P. E., Murray W., Wright M. H., Practical Optimization, Elsevier Academic Press, 84 Theobald's Road, London WC1X 8RR, UK, 2006.

Giton M., Caro-Bretelle A. S., Ienny P., “ Hyperelastic Behaviour Identification by a Forward Problem Resolution: Application to a Tear Test of a Silicone-Rubber", Strain, vol. 42, n 4 , p. 291-297, Nov., 2006.

Grédiac M., “Principe des travaux virtuels et identification”, Compte Rendu de l'Academie des Sciences, vol. 309, p. 1-5, 1989.

Hendricks M., Identification of the mechanical properties of solid materials, $\mathrm{PhD}$ thesis, Eindhoven University of Technology, 1991.

Hoc T., Gélébart L., Crépin J., Zaoui A., “ A procedure for identifying the plastic behavior of single crystals from the local response of polycrystals", Acta Materialia, vol. 51, p. 54775488, 2003.

Kajberg J., Lindkvist G., " Characterization of materials subjected to large strains by inverse modelling based on in-plane displacement fields", International Journal of Solids and Structures, vol. 41, p. 3439-3459, 2004a.

Kajberg J., Sundin K., Melin L., Ståhle P., “ High strain rate tensile and viscoplastic parameter identification using micoscopic high-speed photography”, International Journal of Plasticity, vol. 20, p. 561-575, 2004 b.

Kavanagh K., Clough R., "Finite element applications in the characterization of elastic solids", International Journal of Solids and Structures, vol. 7, p. 11-23, 1971.

Latourte F., Chrysochoos A., Pagano S., Watrisse B., “ Elastoplastic behavior identification for heterogeneous loading and materials", Experimental Mechanics, Special Issue: Inverse Problems, In Press.

Lecompte D., Smits A., Bossuyt S., Sol H., Vantomme J., Van Hemelrijck D., Habraken A., "Quality assessment of Speckle Patterns for Digital Image Correlation", Optics and Lasers in Engineering, vol. 44, p. 1132-1145, November, 2006.

Lecompte D., Smits A., Sol H., Vantomme J., Van Hemelrijck D., “ Mixed numericalexperimental technique for orthotropic parameter identification using biaxial tensile tests on cruciform specimens", International Journal of Solids and Structures, vol. 44, $\mathrm{n}^{\circ} 5$, p. 1643-1656, March, 2007. 
Le Magorou L., Bos F., Rouger F., "Identification of constitutive laws for wood-based panels by means of an inverse method", Composite Science and Technology, vol. 62(4), p. 591-596, 2002.

Lemosse D., Pagnacco E., “ Identification de propriétés matériaux à partir de mesures de champs de déplacements statiques et en présence d'une distribution d'efforts inconnue", Colloque National en Calcul des Structures de Giens, Giens, 2003. cdrom, 15 p.

Linet V., Lepage A., Sol A., Bohineust X., " Validation and Improvement of Body Panels Fe Models From 3d-Shape and Vibration Measurements By Optical Methods", SAE Noise \& Vibration Conference \& Exposition, $\mathrm{n}^{\circ}$ 2001-01-1536, Grand Traverse, MI, USA, April, 2001. cdrom, 8 p.

Mahnken R., " Aspects on the finite-element implementation of the Gurson model including parameter identification", International Journal of Plasticity, vol. 15, n 3-4, p. 1111-1137, September, 1999.

Mahnken R., “ A comprehensive study of a multiplicative elastoplasticity model coupled to damage including parameter identification", Computers and Structures, vol. 74, $\mathrm{n}^{\circ}$ 3-4, p. 179-200, September, 2000.

Mahnken R., Stein E., “ A unified approach for parameter identification of inelastic material models in the frame of the finite element method", Computer Methods in Applied Mechanics and Engineering, vol. 136, n 3-4, p. 225-258, September, 1996.

Mahnken R., Stein E., " Parameter identification for finite deformation elasto-plasticity in principal directions", Computer Methods in Applied Mechanics and Engineering, vol. 147, $\mathrm{n}^{\circ} 3$ 4, p. 17-39, September, 1997.

Meijer R., Douven L., Oomens C., " Characterisation of anisotropic and non-linear behaviour of human skin in-vivo", Comput. Methods in Biomechanics and Biomedical Engineering, vol. 1, p. 13-27, 1997.

Meuwissen M., An inverse method for the mechanical characterisation of metals, $\mathrm{PhD}$ thesis, TU Eindhoven, 1998.

Meuwissen M. H. H., Oomens C. W. J., Baaijens F. P. T., Petterson R., Janssen J. D., “ Determination of the elasto-plastic properties of aluminium using a mixed numerical-experimental method", Journal of Materials Processing Technology, vol. 75, n 1-3, p. 204-211, March, 1998.

Michot S., Cogan S., Foltête E., Raynaud J., Piranda J., “ Apports des techniques de mesures holographiques dans le domaine de la localisation de défauts en dynamique des structures", 2ème Colloque d'Analyse Vibratoire Expérimentale, Blois, 13-14 Novembre, 2001. cdrom, 9 pages.

Molimard J., Le Riche R., Vautrin A., Lee J., “ Identification of the four orthotropic plate stiffnesses using a single open-hole tensile test”, Journal of SEM, vol. 45, n 5 , p. 404-411, 2005.

Montay G., Fran ${ }_{s}$ cois M., Tourneix M., Guelorget B., Vial-Edwards C., Lira I., " Strain and strain rate measurement during the bulge test by elestronic speckle pattern interferometry", Journal of Materials Processing Technology, vol. 184, n 1-3, p. 428-435, April, 2007.

Moreau A., Pagnacco E., Borza D., Lemosse D., “An evaluation of different mixed experimental/numerical procedures using FRF for the identification of viscoelastic materials", International Conference on Noise and Vibration Engineering, ISMA 2006, Leuven, 2006. cdrom, $15 \mathrm{p}$. 
Oomens C., Van Ratingen M., Janssen J., Kok J., Hendriks M., “ A numerical-experimental for a mechanical characterization of biological materials", Journal of Biomechanics, vol. 26(4/5), p. 617-621, 1993.

Padmanabhan S., Hubner J., Kumar A., Ifju P., “ Load and Boundary Conditions Calibration using Full-Field Strain Measurement”, Experimental Mechanics, vol. 46, ${ }^{\circ}$ 5, p. 569-578, 2007.

Pagano S., " A review of identification methods based on full fields measurements", Photomechanics 2006, Int. conf. on full-field measurements techniques and their applications in experimental solid mechanics, 2006.

Pagnacco E., Lemosse D., Borza D., “ A coupled FE based inverse strategy from displacement field measurement subject to an unknown distribution of forces", Photomechanics 2006, Int. conf. on full-field measurements techniques and their applications in experimental solid mechanics, Clermont-Ferrand, France, July 10-12, 2006.

Pagnacco E., Lemosse D., Hild F., Amiot F., “ Inverse strategy from displacement field measurement and distributed forces using FEA", SEM Annual Conference and Exposition on Experimental and Applied Mechanics, June 07-09, 2005. cdrom, 7 p.

Pagnacco E., Moreau A., Lemosse D., “ Inverse strategies for the identification of elastic and viscoelastic material parameters using full-field measurements", Materials Science and Engineering: A, vol. 452-453, p. 737-745, April, 2007.

Piranda J., Foltête E., Raynaud J. L., Michot S., Lepage A., Linet V., “ New trends in modal analysis and model updating using electronic speckle pattern interferometry", in P. Kurka, A. Fleury (eds), X Diname, Ubatuba - SP - Brazil, p. 107-117, 10-14th March, 2003.

Silva G., Le Riche R., Molimard J., Vautrin A., " Exact and Efficient Interpolation using Finite Element Shape Functions", European Journal of Computational Mechanicsp. to appear, October, 2007.

Simon D., Golinval J. C., “ Use of whole-field displacement measurements for model updating of blades", IMAC XXI, n 131, Feb. 3-6, 2003. cdrom, 10 p.

Surell Y., "Les techniques optiques de mesure de champs: essai de classification”, I2M, vol. 4/3-4, p. 11-42, 2004.

Van Ratingen M., Mechanical identification of inhomogeneous solids: a mixed numerical experimental approach, PhD thesis, Eindhoven University of Technology, 1994. 